ADDENDUM

doi:10.1038/nature 19064

\section{Addendum: Non-Joulian magnetostriction}

Harsh Deep Chopra \& Manfred Wuttig

Nature 521, 340-343 (2015); doi:10.1038/nature14459

In this Letter, we showed that the volume of the Fe-Ga crystals we investigated is not conserved in the course of magnetostriction measurements; we termed this phenomenon non-Joulian magnetostriction (NJM), in contrast to Joule magnetostriction, which is volume conserving ${ }^{1}$. We measured NJM in circular-shaped single-crystal disks by applying an in-plane magnetic field and showed that the disks expand radially. Magnetostriction normal to the disks was not reported because we assumed that a negligible vector component of magnetization normal to the disk at fields at which NJM is realized would yield negligible magnetostriction.

Here we present precision measurements undertaken to experimentally verify this assumption. The results are represented by the red curve in Fig. 1. We measured NJM with the strain-gauge technique described in our Letter ${ }^{2}$, which uses a Wheatstone bridge combined with lock-in null detection featuring a resolution of 0.2 p.p.m. We attached microstrain gauges (300- $\mu \mathrm{m}$ gauge length) on the cylindrical surfaces of the samples (lower-right inset in Fig. 1). The data shows a very small strain of 1.3 p.p.m., normal to the disk. In this example, the field was directed parallel to the in-plane [110] axis of the Fe-Ga crystalline disk. Its longitudinal and transverse magnetostriction strains are 70 p.p.m. and 62 p.p.m., respectively, whereas strain along the [100] axis equals 89 p.p.m. (Fig. 1). A similarly negligible strain (1 p.p.m.) occurs when the field is directed along the [100] axis in the plane of the disk (not shown). We also noted that the vector component of magnetization in the [001] direction for an in-plane field (along any in-plane direction) is negligible (upper inset of Fig. 1).

We thus maintain our original conclusion that the disk expands and the volume is not conserved (NJM). In an upcoming paper (R. U. Chandrasena, W. Yang, J. A. Boligitz, M. Forst, A. Scholl, E. Arenholz, F. Kronast, H. Ebert, J. Minár, A. X. Gray \& H.D.C., manuscript in preparation) we show that the observed NJM originates from the nanometre-scale lamellar structure within the highly periodic cellular domains shown in Fig. 3 of our original Letter. Degradation of the lamellar or cellular structure causes the disappearance of non-Joulian behaviour. The generalized Landau-type magnetic structure in Fig. 3 of our original Letter has an electronic origin (charge density waves) and a long coherence. Its existence is a prerequisite of the non-Joulian character of the magnetostriction in $\mathrm{Fe}-\mathrm{Ga}$.

We acknowledge the contribution of C. Jiang, Y. He, P. Stamenov, M. Coey and H. Xu for drawing the omission of this data to our attention. H.D.C. acknowledges the support of National Science Foundation DMR-Condensed Matter Physics grant number 1541236 and Temple University OVPR's Infrastructure Grant and Temple University Merit Scholars grants. M.W. acknowledges the support of ARO grant W911NF-15-1-0615.

1. Joule, J. P. On the effects of magnetism upon the dimensions of iron and steel bars. Phil. Mag. J. Sci. 30, 76-87, 225-241 (1847).

2. Sullivan, M. Wheatstone bridge technique for magnetostriction measurements. Rev. Sci. Instrum. 51, 382 (1980).

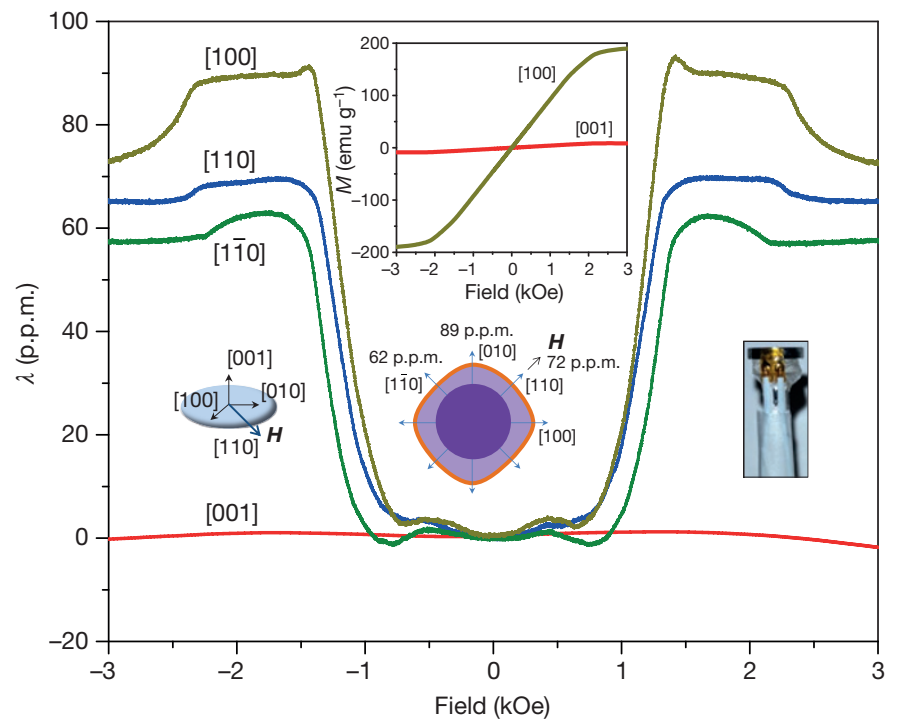

Figure 1 | Volume is not conserved in non-Joulian magnetostriction. Room-temperature magnetostriction $\lambda$ along various principal directions of a slow-cooled $\mathrm{Fe}_{82.9} \mathrm{Ga}_{17.1}$ single crystal with applied field, $\boldsymbol{H}$, along a [110] axis. The red curve shows measured magnetostriction along the [001] direction, this direction being normal to the disk, as shown schematically in the lower-left inset. The lower-middle inset shows expansion along all directions in the plane of the disk. The upper inset shows magnetization $M$ along the [100]-type direction along with simultaneously measured orthogonal (vector) data in the [001] direction, the latter being negligible in the field range for which NJM is observed. The lower-right inset shows a photograph (taken by H.D.C.) of the microstrain gauge setup attached to the cylindrical surface of a sample. 